\title{
Reduction of Intracellular-Reactive Oxygen Species and Diminished Mitogen-Activated Protein Kinases (MAPKs) Activation are Associated with Oral Squamous Cell Carcinoma Cell Aggressiveness
}

\section{Tanyarath UTAIPAN, Apsorn SATTAYAKHOM, Issara PRACHONGSAI, Nurdina CHARONG and Warangkana CHUNGLOK*}

\author{
School of Allied Health Sciences, Walailak University, Nakhon Si Thammarat 80161, Thailand
}

('Corresponding author's e-mail: cwarang@wu.ac.th)

Received: 2 September 2016, Revised: 13 January 2017, Accepted: 22 February 2017

\begin{abstract}
Oral squamous cell carcinoma (OSCC) is a serious health problem in many countries. Several drugs have been used to treat head and neck and oral cavity cancers. However, the success rate has not been impressive because of the heterogeneity of cancerous cells, resulting in differential responsiveness to chemotherapy. Two distinct phenotypes of OSCC cells, the CLS-354/WT and CLS-354/DX cells, have been used as in vitro cell models for this study. CLS-354/DX cells were more aggressive than CLS354/WT cells, supported by the observation that CLS-354/DX cells can undergo epithelial-mesenchymal transition (EMT), grow anchorage-independently, and increase invasiveness. We investigated the preliminary redox status of these 2 cell lines, including levels of reactive oxygen species (ROS) and cellular antioxidants, using flow cytometry analysis and $\mathrm{ABTS}^{+}$free radical scavenging assay, respectively. A 7-fold decrease in ROS level was detected in CLS-354/DX cells, comparing with CLS354/WT cells, while antioxidant capacity was not different from that of CLS-354/WT cells. Hydrogen peroxide, a ROS modulating agent, could induce ROS levels, and caused cell death in CLS-354/WT greater than that of CLS-354/DX cells. Of note, hydrogen peroxide-induced cytotoxicity could be rescued by $N$-acetyl cysteine, confirming ROS-mediated cytotoxicity in both cell lines. ROS-sensitive mitogenactivated protein kinases (MAPKs) were observed using immunoblot assay. The expressions of p-JNK1/2 and p-p38 MAPK in CLS-354/DX cells were absent, while these expressions were abundantly detected in CLS-354/WT cells. This suggests that lower ROS levels, with the concomitant reduction of JNK and p38 MAPK activation in CLS-354/DX cells, are associated with cancer cell aggressiveness. These findings provide significant evidence of the resistance to ROS-modulating agents in aggressive OSCC cells.
\end{abstract}

Keywords: Reactive oxygen species, OSCC cells, mitogen-activated protein kinases

\section{Introduction}

Oral cancer is a subtype of head and neck cancer, which is any cancerous tissue growth located in the oral cavity, and $90 \%$ of cases of this cancer involve oral squamous cell carcinoma (OSCC) [1]. Oral cancer is the tenth most common cancer worldwide, and is one of the top cancer types showing rising mortality rates in many Asian countries [2]. Most patients with OSCC presented pathologic evidence of lymph node metastasis, and a 5-year survival rate is less than $50 \%$ [3]. Oral cancer death often results from local recurrence of regional or systemic metastasis and resistance to therapy [4].

Cellular redox status, a net balance between reduced and oxidized states, is one of the most commonly investigated cellular contexts in the subpopulation of tumor cells, because of a wide range of biological impacts [5]. Reactive oxygen species (ROS), chemically-reactive molecules containing oxygen, act as a secondary messenger, mediating multiple signaling pathways, and play an important role 
in maintaining cellular redox homeostasis [6]. Changes in intracellular ROS levels contribute to a variety of different cellular responses. For instance, high ROS levels can activate cell proliferation, carcinogenesis, and apoptosis [5], whereas low levels of ROS are required for quiescence and selfrenewal [7]. The difference in redox status is associated with aggressiveness of cancer cells and confers drug resistance. More reduced intracellular redox states have been shown to sustain invasive properties and aggressiveness of cancer cells [8,9]. In head and neck squamous carcinoma cells, low ROS levels are used to maintain stemness properties and drug resistance [10]. These collected data indicate that the reduction of ROS may be associated with aggressiveness. A subset of cancer cells with lower ROS levels could be a potential target for more effective therapies.

Mitogen-activated protein kinase (MAPK) signaling pathways are an evolutionary-conserved mechanism of signal transduction in cells involving cell proliferation and apoptosis. Extracellular signalregulated kinase 1/2 (ERK1/2), c-Jun N-Terminal kinase (JNK), and p38 are responsible for each of the signaling transductions [11]. Among these, JNK1/2 and p38 MAPK are very sensitive to oxidative stress induced by ROS [12]. Activation of p38 MAPK with high ROS level is associated with apoptosis induction and attenuation of carcinogenesis [13]. Meanwhile, more aggressive cancer cells have a cytoprotective mechanism through down-regulation of ROS-p38 MAPK signaling [7]. Cisplatin, an ROSgenerating anti-cancer drug, requires p38 MAPK or JNK activation for apoptosis induction [14,15]. Hence, alteration of intracellular ROS in cancer cells may affect the activation of MAPK proteins.

Evidence revealing the association of redox status and the aggressiveness of OSCC cells has not yet been documented. In this study, ROS levels, and the response to ROS-modulating agents, have been performed in 2 cell lines of OSCC; CLS-354/WT (a less aggressive phenotype) and CLS-354/DX (a more aggressive phenotype). We found that the 2 cell lines had different ROS levels, by which CLS-354/DX cells had lower ROS levels and was resistant to hydrogen peroxide-induced toxicity. The reduction of ROS in CLS-354/DX cells was concomitant with decreased phosphorylation of JNK and p38 MAPK. Thus, lower ROS levels might be a mechanism of drug resistance in OSCC cells.

\section{Materials and methods}

\section{Chemicals and reagents}

2,2'-Azino-bis(3-ethylbenzothiazoline-6-sulfonic acid) diammonium salt (ABTS), camptothecin (CPT), dimethyl sulfoxide (DMSO), and 3-(4,5-dimethylthiazol-2-yl)-2,5-diphenyltetrazolium bromide (MTT), and $N$-acetyl-cysteine (NAC) were purchased from Sigma-Aldrich Corp., (St. Louis, MO, USA). Fetal bovine serum (FBS) was obtained from Biochrom GmbH (Berlin, Germany). RPMI-1640, phosphate buffer saline (PBS), and penicillin/streptomycin were purchased from PAA Laboratories $\mathrm{GmbH}$ (Pasching, Austria). 2',7'-dichlorodihydrofluorescein diacetate ( $\left.\mathrm{H}_{2} \mathrm{DCFDA}\right), 0.25 \%$ trypsinEDTA, and stable L-glutamine were purchased from Gibco, Life Technologies (Carlsbad, CA, USA). Becto agar was purchased from BD Biosciences (San Jose, CA, USA). ECL Plus Western blotting Detection Reagent was purchased from Amersham Biosciences Corp., (Piscataway, NJ, USA). Antibodies for vimentin, E-cadherin, MAPKs, and $\beta$-actin, and HRP-linked secondary antibodies, were purchased from Cell Signaling Technology, Inc., (Danvers, MA, USA).

\section{Cell culture}

The fibroblast-like phenotype of the human mouth carcinoma cell line, CLS-354/DX, and the epithelium-like phenotype, CLS-354/WT (passage number 35 - 45) were used as in vitro cell models for this study. These 2 cell lines were established from the original human mouth carcinoma cell line CLS354 (Cell Line Service, Germany) [16]. CLS-354/WT and CLS-354/DX cells were cultured in RPMI1640 medium supplemented with $10 \%$ fetal bovine serum (FBS), $1 \%$ penicillin/streptomycin, and $2 \mathrm{mM}$ stable L-glutamine. For EMT study, less-transformed, epithelium-like (EPI) cells and more-transformed, fibroblast-like (FIB) cells (gifts from Assoc. Prof. Dr. Walee Chamulitrat, University Heidelberg Hospital, Heidelberg, Germany) were cultured in complete DMEM medium. All cell lines were maintained in an atmosphere of $95 \%$ humidity and $5 \% \mathrm{CO}_{2}$ at $37{ }^{\circ} \mathrm{C}$. 


\section{Anchorage-independent growth assay}

CLS-354/WT and CLS-354/DX cells were tested for anchorage-independent growth using the method described previously [17]. Briefly, a $0.33 \%$-agarose mixture containing cells was overlaid onto the prepared $0.5 \%$-agar bottom layer. Cells were plated at a density of $7.1 \times 10^{3}$ cells $/ \mathrm{cm}^{2}$. Agar plates were kept in a $5 \%-\mathrm{CO}_{2}$ incubator at $37{ }^{\circ} \mathrm{C}$ and were fed with RPMI-1640/10\% FBS every 5 days. Colonies $(>20$ cells/colony) were counted in the third week after plating under a phase-contrast microscope. The number of colonies was normalized to a $\%$ based on the total number of seeded cells as percent efficiency.

\section{Invasion assay}

Cell invasion assay was determined using the Cell Invasion Assay Kit (ECM550, Chemicon, USA) according to the manufacturer's instructions. Briefly, cell suspensions of CLS-354/WT or CLS-354/DX at a density of $5 \times 10^{5}$ cells $/ \mathrm{ml}$ were seeded in serum-free RPMI-1640 into the upper chamber and allowed to invade towards media containing $10 \% \mathrm{FBS}$ as a chemo-attractant in the lower chamber for $24 \mathrm{~h}$ in a $\mathrm{CO}_{2}$ incubator. After incubation, invasive cells on the lower surface of the membrane were stained with staining solution for $20 \mathrm{~min}$. The invasive cells were then photographed using an inverted microscope, with at least 3 random views. To quantitate the invasive cells, the stained invasive cells on the lower surface of the membrane were dissolved with $10 \%$ acetic acid. The absorbance of the solution was determined at $560 \mathrm{~nm}$.

\section{Detection of intracellular ROS}

CLS-354/WT and CLS-354/DX cells were seeded in 6-well plate $\left(4.2 \times 10^{4} \mathrm{cells} / \mathrm{cm}^{2}\right)$ and allowed to grow for $48 \mathrm{~h}$. For the analysis of hydrogen peroxide $\left(\mathrm{H}_{2} \mathrm{O}_{2}\right)$-induced ROS, cells were treated with $\mathrm{H}_{2} \mathrm{O}_{2}$ $(400 \mu \mathrm{M})$ or in combination with $N$-acetyl cysteine (NAC) $(5 \mathrm{mM})$ for $24 \mathrm{~h}$. Treated cells were then harvested, washed with PBS, and incubated in PBS containing $10 \mu \mathrm{M} \mathrm{H}_{2}$ DCFDA for $30 \mathrm{~min}$ at $37{ }^{\circ} \mathrm{C}$ in dark conditions. After incubation, cells were washed, re-suspended with PBS, and subjected to a flow cytometer for analysis of ROS production. The mean fluorescence intensity (MFI) of the green fluorescence (FL1) obtained from 20,000 cells was quantitated using CellQuest ${ }^{\mathrm{TM}}$ Pro software (BD Biosciences, San Jose, CA, USA).

\section{Measurement of total antioxidant capacity}

The cellular total antioxidant capacity was performed by using ABTS cation radical (ABTS ${ }^{*}$ ) scavenging assay. Cells were harvested and lysed in sonication buffer $(150 \mathrm{mM}$ Tris- $\mathrm{HCl}, \mathrm{pH} 7.8,10 \mathrm{mM}$ PMSF, $0.2 \mathrm{mM} \mathrm{Na} \mathrm{VO}_{4}, 0.01 \mathrm{M} \mathrm{NaF}, 0.014 \mathrm{mM}$ chymostatin, $99.3 \mathrm{KU} / \mathrm{ml}$ aprotinin, and $0.01 \mathrm{mg} / \mathrm{ml}$ pepstatin A) using a sonicator. Bradford's reagent kit was used to measure protein concentration of the cell lysates. The cell lysates of $0.5 \mathrm{mg} / \mathrm{ml}$ protein were mixed with working ABTS ${ }^{+}$solution and incubated in dark conditions for $3 \mathrm{~min}$. Then, the absorbance was read at $734 \mathrm{~nm}$ using a microplate reader. The standard curve was linear, using Trolox between $6.25-250 \mu \mathrm{M}$. The unit of cellular total antioxidant capacity was defined as Trolox equivalent antioxidant capacity (TEAC) per $1 \mathrm{mg}$ protein.

\section{Cytotoxicity test}

CLS-354/WT and CLS-354/DX cells were seeded into the 96-well plates at a density of $4.7 \times 10^{4}$ cells $/ \mathrm{cm}^{2}$ and allowed to grow for $48 \mathrm{~h}$. The cells were then treated with various concentrations of $\mathrm{H}_{2} \mathrm{O}_{2}$ $(0-1000 \mu \mathrm{M})$ with or without NAC $(0.6-10 \mathrm{mM})$ for $24 \mathrm{~h}$ at $37{ }^{\circ} \mathrm{C}$ in a humidified $5 \% \mathrm{CO}_{2}$ atmosphere. After treatment, spent media were removed and replaced with complete medium overnight. Cell viability was performed by MTT assay, based on the conversion of MTT to formazan crystals by mitochondrial dehydrogenases from viable cells. MTT was dissolved in cultured medium to obtain a final concentration of $0.5 \mathrm{mg} / \mathrm{ml}$ and added into each well. After incubation at $37{ }^{\circ} \mathrm{C}$ in $\mathrm{CO}_{2}$ incubator for $3 \mathrm{~h}$, formazan crystals were dissolved with DMSO. Absorbance was detected at $560 \mathrm{~nm}$, with subtraction of background at $670 \mathrm{~nm}$, by using a microplate reader. The percentage of cell viability (\%) was calculated using the following equation; 
http://wjst.wu.ac.th

$\left[\left(\mathrm{Abs}_{\text {treated sample }} / \mathrm{Abs}\right.\right.$ untreated sample $\left.) \times 100\right]$.

Western blot analysis

CLS-354/WT and CLS-354/DX cells were cultured in a complete medium at $\sim 80 \%$ confluence. After $48 \mathrm{~h}$, the cells were harvested and lysed in sonication buffer containing protease inhibitors by using a sonicator. Cell lysates containing equal amount of protein $(30 \mu \mathrm{g})$ were then prepared in Laemmli sample buffer. The protein samples were separated in a $10 \%$ SDS-PAGE gel and transferred onto a PVDF membrane. The membrane was blocked with $5 \%$ non-fat dry milk, washed with TBTS, and probed with primary antibodies against vimentin (1:2500), E-cadherin (1:5000), phospho-ERK1/2 (1:1000), phospho-JNK1/2 (1:1000), phospho-p38 (1:1000), total ERK1/2 (1:1000), total JNK (1:500), total p38 (1:1000), and $\beta$-actin (1:5000) at $4{ }^{\circ} \mathrm{C}$ overnight. The blots were probed with secondary antirabbit antibody (1:5000) at RT for $1 \mathrm{~h}$. Subsequently, the membranes were washed, and proteins were visualized using an ECL Western blot detection system.

\section{Statistical analysis}

All experiments were performed at least 3-independent times. The results were expressed as the mean \pm standard error of mean (SEM). Statistical analysis was determined by Student t-test or one-way ANOVA, followed by multiple comparison tests using GraphPad Prism 6 (GraphPad Software Inc., La Jolla, CA, USA). Differences of $p<0.05$ were considered significant.

\section{Results and discussion}

EMT-like phenotype, anchorage-independent growth, and invasiveness of CLS-354/DX cells

CLS-354 cells are human oral squamous cell carcinoma (OSCC), originally characterized as an epithelial cell type. In this study, some cells at passage number 27 spontaneously exhibited a fibroblastlike morphology (elongated shape) after several subcultures, resulting in the appearance of 2 distinct phenotypes an epithelium-like phenotype, CLS-354/WT, and a fibroblast-like phenotype, CLS-354/DX (Figure 1A). These 2 phenotypes of the cells were separated using differential trypsinization, by which CLS-354/DX cells were more easily detached than CLS-354/WT cells [16]. The morphology of CLS354/DX cells was stable upon culturing in a complete RPMI-1640 with $10 \%$ FBS. These cells did not reverse back to an epithelium-like appearance.

To confirm the acquisition of epithelial-mesenchymal transition (EMT), we further studied the expression of EMT-related proteins, including E-cadherin and vimentin in CLS-354/DX cells. In addition, epithelium-like transformed human gingival keratinocytes (EPI cells) and fibroblast-like transformed human gingival keratinocytes (FIB cells) were used as positive controls for partial EMT- and EMT-like cells, respectively. As shown in Figure 1B, E-cadherin (a marker protein of an epithelial cell) was strongly expressed in CLS-354/WT cells, similar to that of EPI cells, but not in CLS-354/DX and FIB cells. Conversely, vimentin (a marker protein of a mesenchymal cell) was highly expressed in CLS354/DX cells, approximately equal to that of FIB cells. CLS-354/WT and EPI cells partially expressed vimentin, suggesting a partial EMT-like phenotype. The expression of these 2 proteins indicates that CLS-354/DX cells acquired EMT properties, while partial acquisition of EMT phenotype was observed in CLS-354/WT cells. This phenomenon has been linked to the EMT process, which is a biological process allowing a polarized epithelial cell to undergo multiple changes towards mesenchymal cells [18]. Cancer cells that undergo EMT lose their adherent properties, and can be easily detached from the extracellular matrix compartment [19]. 
A

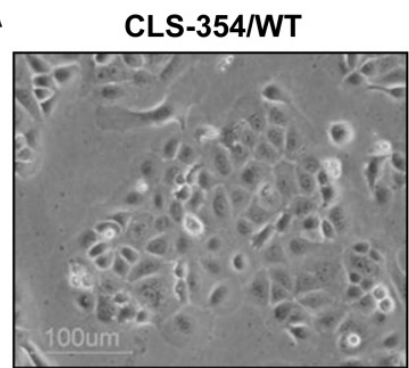

C

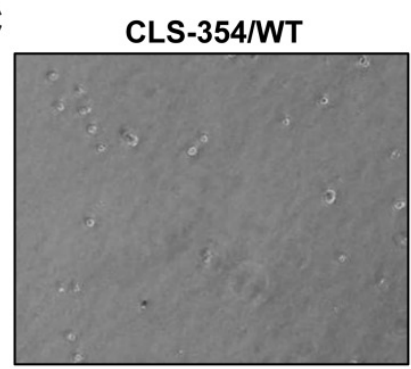

E

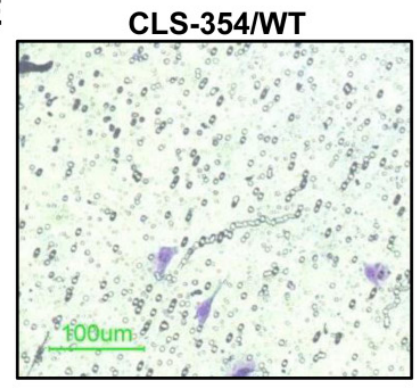

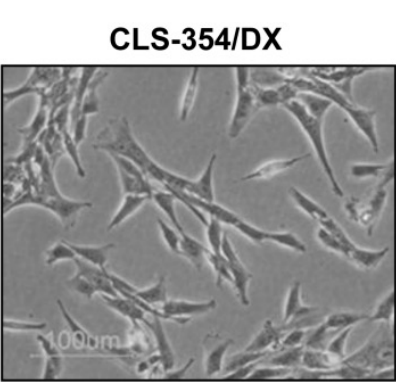

CLS-354/DX
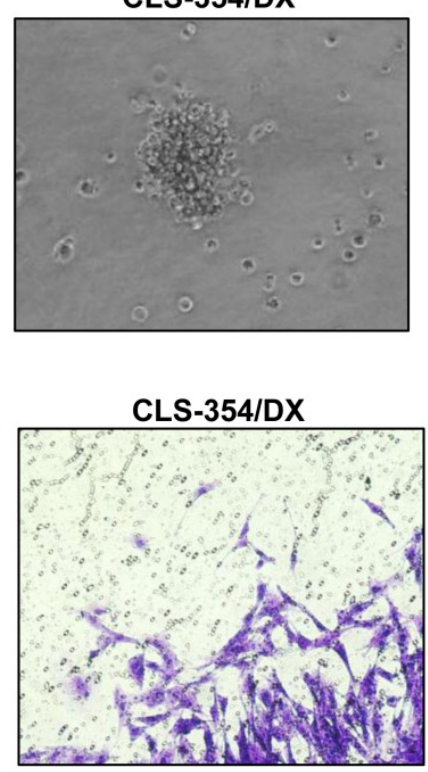

B

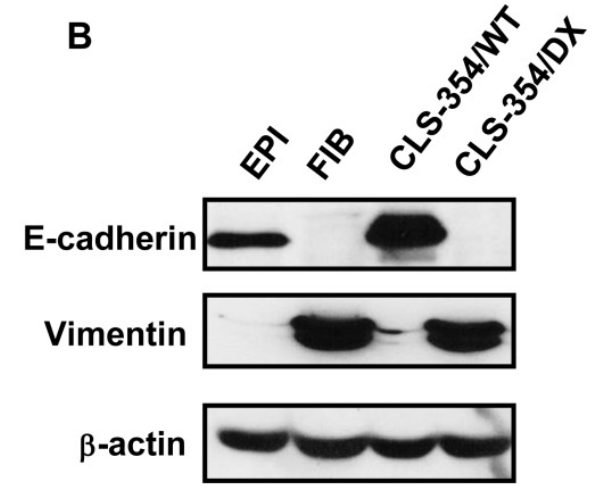

D

\begin{tabular}{|cc|}
\hline Cell lines & $\begin{array}{c}\text { AIG efficiency } \\
(\%)\end{array}$ \\
\hline CLS-354/WT & $0.00 \pm 0.000$ \\
CLS-354/DX & $0.021 \pm 0.004$ \\
\hline
\end{tabular}

F

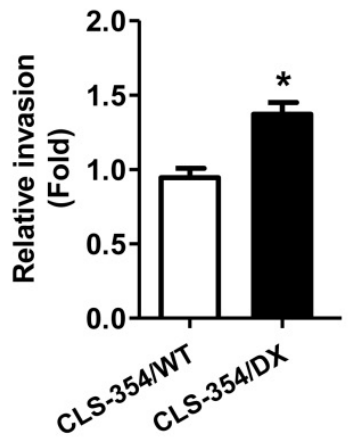

Figure 1 The acquisition of EMT in CLS-354/DX cells. (A) The cell morphology of CLS-354/WT and CLS-354/DX under a phase contrast microscope $(\times 100$ magnification). (B) E-cadherin and vimentin expression by Western blot in EPI, FIB, CLS-354/WT, and CLS-354/DX cells (C) Colony formation in semi-solid agar of CLS-354/WT (left) and CLS-354/DX cells (right). (D) The efficiency of anchorageindependent growth (AIG) was determined by the normalization of the number of colonies to the total number of seeded cells. Data were expressed as mean \pm SEM $(n=4)$. (E) Invasion ability and $(F)$ quantitative analysis of the relative invasion of CLS-354/WT and CLS-354/DX cells. Data were expressed as mean $\pm \operatorname{SEM}(\mathrm{n}=5) ;{ }^{*} p<0.005$ vs. CLS-354/WT.

EMT is often seen in advanced-stage cancer in order to confer aggressive behaviors, such as metastasis and chemoresistance [20]. To compare the degree of aggressiveness among CLS-354/WT and CLS-354/DX cells, the in vitro anchorage-independent growth ability was carried out by colony formation assay in soft agar. As shown in Figure 1C, right, CLS-354/DX cells grew anchorageindependently, while CLS-354/WT cells was unable to form colonies (Figure 1C, left). The colonyforming efficiency of CLS-354/DX cells was $0.021 \%$ (Figure 1D). Anchorage-independent growth is a 
http://wjst.wu.ac.th

characteristic of cell transformation for detecting the malignant transformation of cells [21]. Concomitantly, an increasing number of invaded cells in CLS-354/DX cells through a matrigel-coated membrane were observed and photographed (Figure 1E). Quantitative analysis of the relative invasion of CLS-354/DX cells was significantly higher than CLS-354/WT cells (Figure 1F). Thus, CLS-354/DX cells are capable of gaining aggressiveness, indicating a higher degree of malignancy than CLS-354/WT cells.

\section{Lower ROS levels in CLS-354/DX cells}

Changes in ROS levels, either by endogenous cell metabolism or by exogenous oxidants, affect cellular redox status [5]. To evaluate preliminary oxidative and antioxidant profiling, intracellular ROS and total antioxidant capacity were measured to represent oxidant/antioxidant balance in our cell model. The results showed that basal intracellular ROS levels in CLS-354/WT cells were significantly greater than that of CLS-354/DX ( 7-fold) (Figure 2A). However, these 2 cells showed no difference in total antioxidant capacity (Figure 2B). Thus, ROS formation between CLS-354/WT and CLS-354/DX cells was distinctly different. It has been suggested that endogenous ROS levels can be modified in different degrees of malignancy [8,9]. The elevation or reduction of endogenous ROS levels may be affected by the changes in cell metabolism and the modulation of the antioxidant system. For instance, NADPH oxidase 1 (NOX1), a major ROS generator in epithelial cells, has been found to be overexpressed in cancer cells and involved in increasing ROS formation, leading to the contribution of neoplastic formation in epithelial cancer [22]. Defects in antioxidant enzyme systems have been reported in OSCC biopsies [23]. On the other hand, advanced malignant cells, such as cancerous stem-like cells, can adapt themselves by increasing antioxidant glutathione $(\mathrm{GSH})$ to defend against ROS. The reduction of ROS has been suggested to promote cancer cell survival, therapy resistance, and metastatic progression [7]. Although antioxidants in our cell model have not yet been fully investigated, measurement of antioxidant systems and cancer stem cell markers will be carried out to provide valuable clues in further experiments.

\section{A}

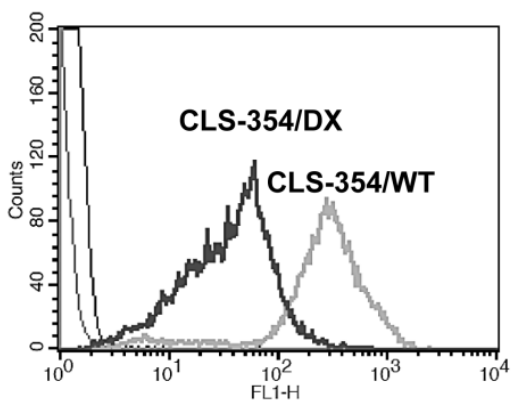

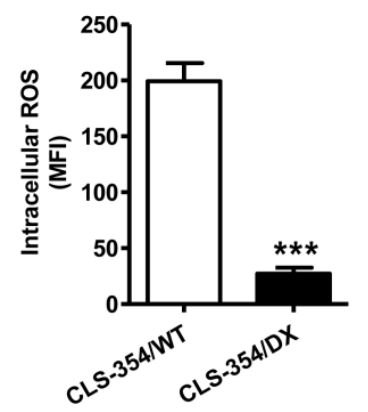

B

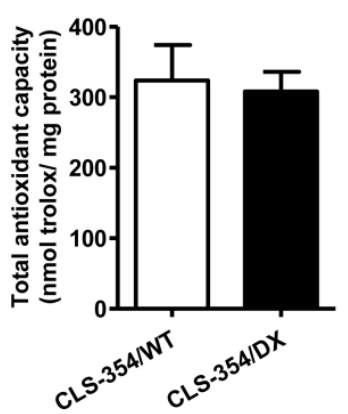

Figure 2 Intracellular reactive oxygen species (ROS) and total antioxidant levels in CLS-354/WT and CLS-354/DX cells. (A) Intracellular ROS of cells was determined by $\mathrm{H}_{2}$ DCFDA fluorescent probe and analysed by flow cytometric approaches. The fluorescence signal, which was proportional to intracellular ROS, was represented as a histogram of ROS (left) and mean florescent intensities (MFI) (right). (B) Total antioxidant capacity in cell lysate of CLS-354/WT and CLS-354/DX cells was determined by ABTS radical cation decolorization assay. Data were expressed as mean \pm SEM from 3-independent experiments; $* * * p<0.001$ vs. CLS-354/WT. 
A

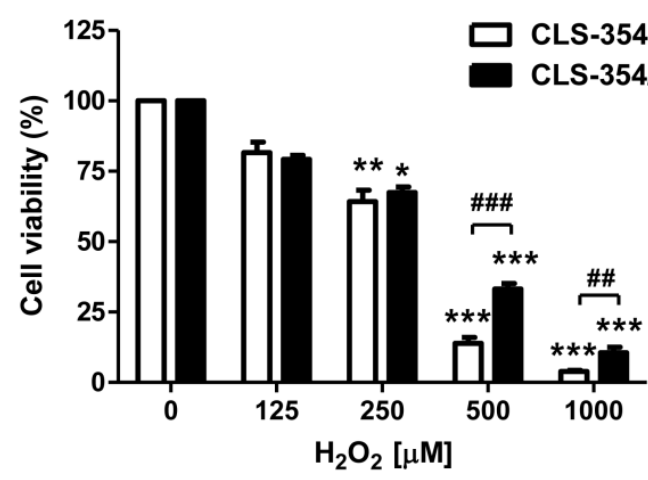

C

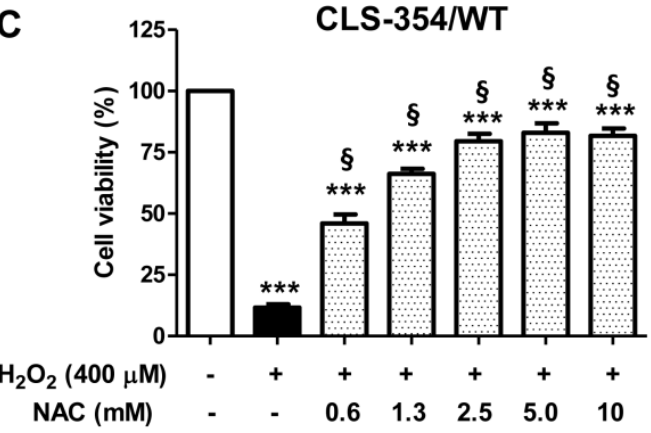

E
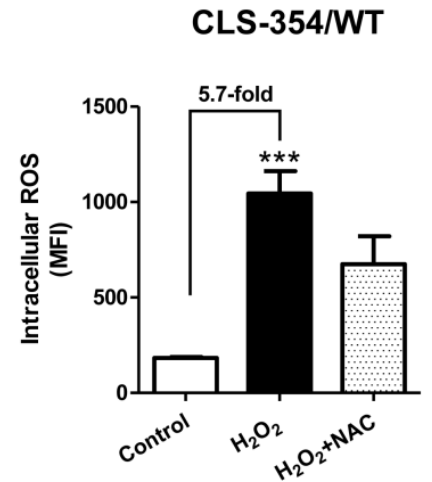

B

\begin{tabular}{|cc|}
\hline Cell lines & $\mathrm{IC}_{50}(\mu \mathrm{M})$ of $\mathrm{H}_{2} \mathrm{O}_{2}$ \\
\hline CLS-354/WT & $258.3 \pm 7.26$ \\
CLS-354/DX & $378.3 \pm 13.02$ \\
\hline
\end{tabular}

D

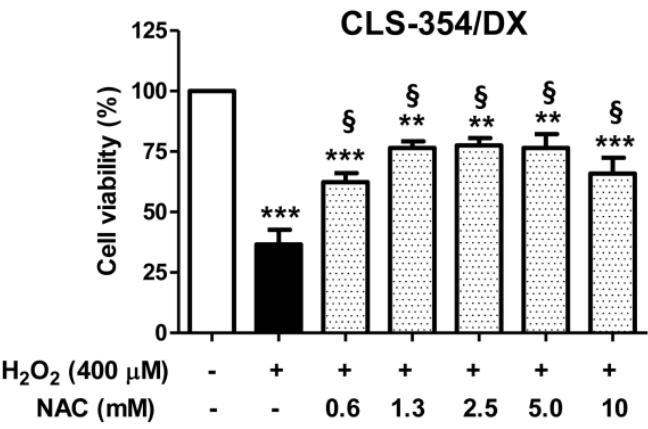

$\mathbf{F}$

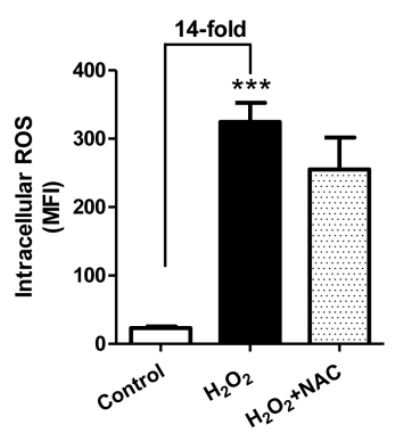

Figure 3 Responsiveness to hydrogen peroxide of CLS-354/WT and CLS-354/DX cells. (A) CLS354/WT and CLS-354/DX cells were treated with hydrogen peroxide $\left(\mathrm{H}_{2} \mathrm{O}_{2} ; 125-1000 \mu \mathrm{M}\right)$. (B) $\mathrm{IC}_{50}$ values upon $\mathrm{H}_{2} \mathrm{O}_{2}$ treatment were determined from the $\mathrm{H}_{2} \mathrm{O}_{2}$ response curve. (C) CLS-354/WT cells and (D) CLS-354/DX cells were treated with $\mathrm{H}_{2} \mathrm{O}_{2}(400 \mu \mathrm{M})$ in the presence of $N$-acetyl-cysteine (NAC, $0.6-10 \mathrm{mM}$ ). After $24 \mathrm{~h}$ treatment, the cell viability was assessed by MTT assay. (E-F) Intracellular ROS levels upon treatment with $\mathrm{H}_{2} \mathrm{O}_{2}(400 \mu \mathrm{M})$ with or without NAC $(5 \mathrm{mM})$ in (E) CLS-354/WT and (F) CLS-354/DX cells were determined using flow cytometry analysis. Data are expressed as mean \pm SEM from at least 3 independent experiments; ${ }^{*} p<0.05,{ }^{* *} p<0.01$, ${ }^{* * *} p<0.001$ vs. untreated control; \#\# $p$ $<0.01$, \#\#\# $p<0.001$ vs. CLS-354/WT; $\S p<0.001$ vs. $\mathrm{H}_{2} \mathrm{O}_{2}$ alone. 


\section{Lesser responsiveness to ROS-modulating agent in CLS-354/DX cells}

Hydrogen peroxide $\left(\mathrm{H}_{2} \mathrm{O}_{2}\right)$, a potent ROS-modulating agent, was adopted in this cell model. Cell viability was determined following $\mathrm{H}_{2} \mathrm{O}_{2}$ treatment for $24 \mathrm{~h}$ using MTT assay. The results showed that $\mathrm{H}_{2} \mathrm{O}_{2}$ in concentrations ranging from 250 to $1000 \mu \mathrm{M}$ decreased cell viability in a dose-dependent manner in both cell lines (Figure 3A). Notably, CLS-354/DX cells exhibited lower response to $\mathrm{H}_{2} \mathrm{O}_{2}$ than that of CLS-354/WT cells at 500 and $1000 \mu \mathrm{M}$ (Figure 3A). The half maximal inhibitory concentrations $\left(\mathrm{IC}_{50}\right)$ upon $\mathrm{H}_{2} \mathrm{O}_{2}$ treatment in CLS-354/WT and CLS-354/DX cells were 258.30 $\pm 7.26 \mu \mathrm{M}$ and $378.30 \pm 13.02$ $\mu \mathrm{M}$, respectively, which were significantly different (Figure 3B). These data indicate that CLS-354/DX cells are more resistant to $\mathrm{H}_{2} \mathrm{O}_{2}$-induced cell death than CLS-354/WT cells.

To confirm the induction of intracellular ROS-mediated cell death upon $\mathrm{H}_{2} \mathrm{O}_{2}$ treatment in both cell lines, the combined treatment of $\mathrm{H}_{2} \mathrm{O}_{2}$ with NAC, a specific ROS scavenger, was then performed. NAC at different concentrations, ranging from 0.6 to $10 \mathrm{mM}$, could improve cell viability in the presence of $400 \mu \mathrm{M} \mathrm{H}_{2} \mathrm{O}_{2}$ in both cell lines (Figures 3C and 3D). $\mathrm{H}_{2} \mathrm{O}_{2}$-induced ROS production in the presence or absence of NAC was confirmed again by flow cytometry analysis. ROS production was increased 5.7fold and 14-fold in CLS-354/WT and CLS-354/DX cells, respectively. Moreover, 5 mM NAC could protect against ROS production (Figures 3E and 3F), indicating the existence of intracellular ROS upon $\mathrm{H}_{2} \mathrm{O}_{2}$ treatment.

Anticancer drugs mediate apoptotic cell death in various tumor cells via ROS generation. ROSinduced apoptosis could be attenuated by using NAC, indicating the role of an ROS-mediated mechanism [15]. Differential redox status in gastrointestinal cancer cells [7] and head and neck cancer cells [10] affected anticancer drug responsiveness. These cancer cells exhibited lower ROS levels, and they were found to be more resistant to anticancer drugs than other cancer cells with high ROS levels [7,10]. Studies demonstrated that cancer cells expressing lower ROS levels are more resistant to exogenous pro-oxidant compounds $[9,24]$. Our results suggest that lower basal ROS levels in CLS-354/DX cells are likely to be involved in resistance to ROS-modulating agent. The different response mechanisms might be related to the difference of a cell's antioxidative capability, such as intracellular GSH content [7] and antioxidant enzyme levels [23], thus requiring further investigation.

\section{Reduction of MAPK phosphorylation in CLS-354/DX cells}

We determined the expression of phosphorylated MAPK, including p-ERK1/2, p-JNK1/2, p-p38, and their total forms, by Western blot. The fully phosphorylated form of MAPKs was shown when the cells reached approximately $80 \%$ confluence [25], similar to our observation in Figure 4. We also observed a decrease of total forms, especially total-ERK and total-JNK, when fully phosphorylated forms were activated in highly confluent conditions. Therefore, expression of phosphorylated MAPKs can be compared with endogenous control $\beta$-actin rather than total forms. The both cell lines expressed pERK1/2, but with different molecular size (Figure 4, left). A fully phosphorylated ERK in these cells may mostly depend on MEK activation. Autocrine-signaling through growth factors would result in MEK/ERK activation and cell proliferation [26]. The differential pattern of ERK phosphorylation may be due to an ERK1 mutant, which could increase the threonine phosphorylation (ERK1) with an increase in its activation [27]. Interestingly, the expressions of p-JNK1/2 and p-p38 in CLS-354/DX cells were low to undetectable (Figure 4, middle and right). It is suggested that the phosphorylated JNK1/2 and p38 are increased in parallel with high ROS levels. In particular, JNK1/2 and p38 MAPK are redox-sensitive kinases, which are responsible for apoptosis activation in cancer cells [12]. Differential intracellular ROS levels in cancer cells have been reported to confer p38 MAPK phosphorylation. In more aggressive phenotypes of gastric cancer cells, it was reported that the ablation of ROS-p38 MAPK is caused by a CD44 variant. This variant in those aggressive cells promoted glutathione (GSH) synthesis via interaction with glutamate-cystine transporter $\mathrm{xCT}$. This mechanism contributed to the reduction of ROS levels and, consequently, decreased p38 MAPK phosphorylation, a downstream target of ROS [7]. On the other hand, high ROS levels observed in tumor cells have been shown to involve apoptosis induction via p38 MAPK activation in order to inhibit tumor formation and carcinogenesis [13]. Upon treatment with ROSmodulating compounds, several studies demonstrated that JNK1/2 and p38 MAPK are required for ROS- 
http://wjst.wu.ac.th

induced apoptosis in oral and laryngeal cancer cells. The abrogation of JNK1/2 and p38 using specific inhibitors can protect ROS-mediated apoptosis, indicating their crucial role in ROS response [28,29]. Apoptosis-inducing activity of anticancer drug cisplatin also relates with ROS-mediated p38 MAPK and JNK activation $[14,15]$. JNK1/2 and p38 MAPK may likely be key ROS effectors in our cell model. This study suggests that lower ROS levels in CLS-354/DX cells are associated with the ablation of JNK1/2 and p38 MAPK, which might, in part, lead to resistance in ROS-modulating agent-induced cell death.
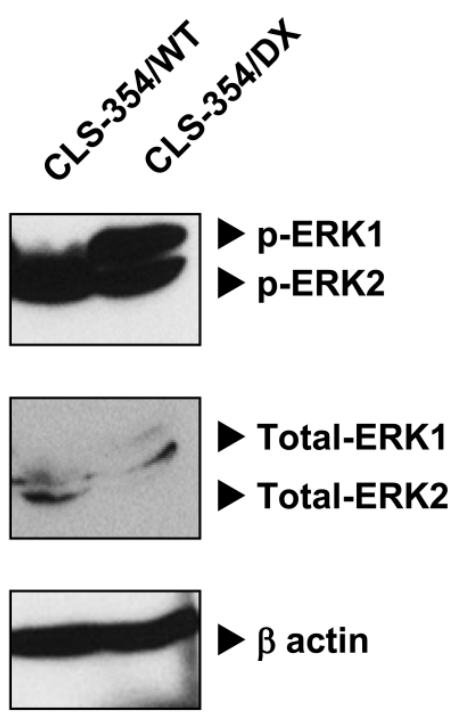
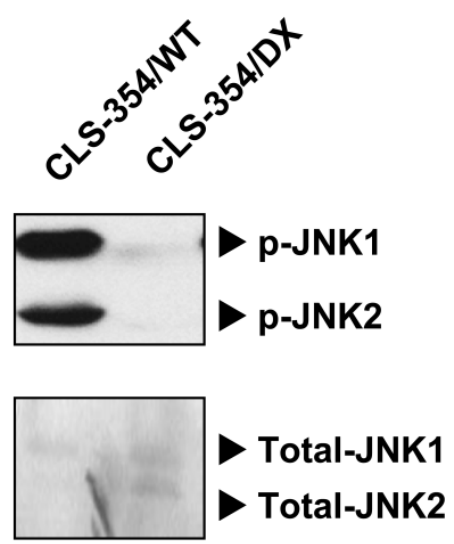

$\beta$ actin
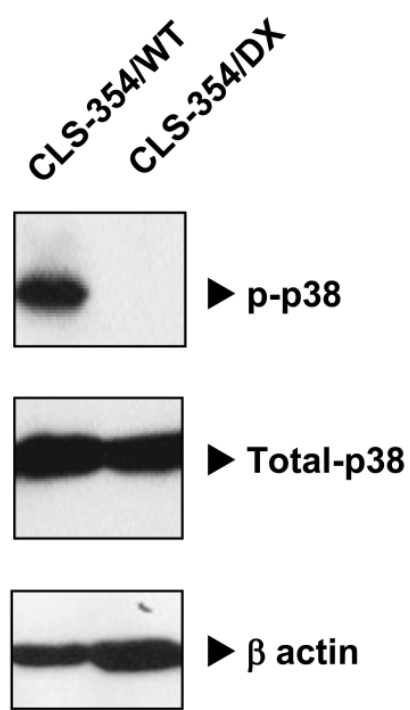

Figure 4 Expression of MAPKs in CLS-354/WT and CLS-354/DX cells. The protein expression of p-ERK1/2, p-JNK1/2, p-p38, and their total forms was observed by Western blot. $\beta$-actin was detected as an internal loading control. The present data was representative of 3-independent experiments.

\section{Conclusions}

We have demonstrated that OSCC cells exhibited heterogeneity after several subcultures. Some cells had changed to an EMT-derived phenotype (CLS-354/DX) which were more aggressive and had much lower levels of ROS than the epithelium-like CLS-354/WT cells. Lower ROS levels in CLS354/DX cells conferred ROS-modulating agent resistance, with concomitant loss of JNK1/2 and p38 activation. These findings are informative of the concept that distinct ROS levels in subpopulations of cancer cells affect sensitivity to ROS-modulating compounds in advanced OSCC cells.

\section{Acknowledgements}

This work was supported by the program of strategic scholarships fellowships frontier research networks for the Ph.D. sandwich program doctoral degree from the Office of the Higher Education Commission (OHEC), Thailand (06/2556); Walailak University (WU56113 and WU59201), and the Walailak University Fund for graduate studentship (27/2556 and WU55603). 
http://wjst.wu.ac.th

\section{References}

[1] J Massano, FS Regateiro, G Januário and A Ferreira. Oral squamous cell carcinoma: Review of prognostic and predictive factors. Oral Surg Oral Med. Oral Pathol. Oral Radiol. Endod. 2006; 102, 67-76.

[2] S Warnakulasuriya. Global epidemiology of oral and oropharyngeal cancer. Oral Oncol. 2009; 45, 309-16.

[3] X Zhang,Y Liu, MZ Gilcrease,XH Yuan, GL Clayman, K Adler-Storthz and Z Chen. A lymph node metastatic mouse model reveals alterations of metastasis-related gene expression in metastatic human oral carcinoma sublines selected from a poorly metastatic parental cell line. Cancer 2002; 95, 1663-72.

[4] L Olasz, E Orsi, T Markó and J Szalma. Induction chemotherapy response and recurrence rates in correlation with N0 or N+ stage in oral squamous cell cancer (OSCC). Cancer Metastasis Rev. 2010; 29, 607-11.

[5] JM Matés, JA Segura, FJ Alonso and J Márquez. Intracellular redox status and oxidative stress: Implications for cell proliferation, apoptosis, and carcinogenesis. Arch. Toxicol. 2008; 82, 273-99.

[6] D Trachootham, W Lu, MA Ogasawara, NRD Valle and P Huang. Redox regulation of cell survival. Antioxid. Redox Signal 2008; 10, 1343-74.

[7] T Ishimoto, O Nagano, T Yae, M Tamada, T Motohara, H Oshima, M Oshima, T Ikeda, R Asaba, H Yagi, T Masuko, T Shimizu, T Ishikawa, K Kai, E Takahashi, Y Imamura, Y Baba, M Ohmura, M Suematsu, H Baba and H Saya. CD44 variant regulates redox status in cancer cells by stabilizing the xCT subunit of system xc(-) and thereby promotes tumor growth. Cancer Cell. 2011; 19, 387-400.

[8] L Chaiswing, W Zhong, Y Liang, DP Jones and TD Oberley. Regulation of prostate cancer cell invasion by modulation of extra- and intracellular redox balance. Free Radic. Biol. Med. 2012; 52, 452-61.

[9] L Chaiswing, JM Bourdeau-Heller, W Zhong and TD Oberley. Characterization of redox state of two human prostate carcinoma cell lines with different degrees of aggressiveness. Free Radic. Biol. Med. 2007; 43, 202-15.

[10] CW Chang, YS Chen, SH Chou, CL Han, YJ Chen, CC Yang, CY Huang and JF Lo. Distinct subpopulations of head and neck cancer cells with different levels of intracellular reactive oxygen species exhibit diverse stemness, proliferation, and chemosensitivity. Cancer Res. 2014; 74, 6291305.

[11] JY Fang and BC Richardson. The MAPK signaling pathways and colorectal cancer. Lancet Oncol. $2005 ; 6,322-7$.

[12] X Sui, N Kong, L Ye, W Han, J Zhou, Q Zhang, C He and H Pan. p38 and JNK MAPK pathways control the balance of apoptosis and autophagy in response to chemotherapeutic agents. Cancer Lett. 2014; 344, 174-9.

[13] I Dolado, A Swat, N Ajenjo, G De Vita, A Cuadrado and AR Nebreda. p38 $\alpha$ MAP kinase as a sensor of reactive oxygen species in tumorigenesis. Cancer Cell. 2007; 11, 191-205.

[14] P Bragado, A Armesilla, A Silva and A Porras. Apoptosis by cisplatin requires p53 mediated p38 $\alpha$ MAPK activation through ROS generation. Apoptosis 2007; 12, 1733-42.

[15] L Pereira, A Igea, B Canovas, I Dolado and AR Nebreda. Inhibition of p38 MAPK sensitizes tumour cells to cisplatin-induced apoptosis mediated by reactive oxygen species and JNK. EMBO Mol. Med. 2013; 5, 1759-74.

[16] T Utaipan, A Athipornchai, A Suksamrarn, S Chunsrivirot and W Chunglok. Isomahanine induces endoplasmic reticulum stress and simultaneously triggers p38 MAPK-mediated apoptosis and autophagy in multidrug-resistant human oral squamous cell carcinoma cells. Oncol. Rep. 2017; 37, 1243-52.

[17] W Chunglok, W Ittarat, P Tomakidi, R Schmidt, W Stremmel and W Chamulitrat. Human gingival mucosal keratinocytes exhibiting anchorage-independent growth express increased inducible nitric oxide synthase: regulation by MAP kinases. Nitric Oxide. 2004; 11, 237-46. 
http://wjst.wu.ac.th

[18] R Kalluri and RA Weinberg. The basics of epithelial-mesenchymal transition. J. Clin. Invest. 2009; 119, 1420-8.

[19] C Morata-Tarifa, G Jiménez, MA García, JM Entrena, C Griñán-Lisón, M Aguilera, M Picon-Ruiz and JA Marchal. Low adherent cancer cell subpopulations are enriched in tumorigenic and metastatic epithelial-to-mesenchymal transition-induced cancer stem-like cells. Sci. Rep. 2016; 6, 18772.

[20] A Singh and J Settleman. EMT, cancer stem cells and drug resistance: an emerging axis of evil in the war on cancer. Oncogene 2010; 29, 4741-51.

[21] SI Shin, VH Freedman, R Risser and R Pollack. Tumorigenicity of virus-transformed cells in nude mice is correlated specifically with anchorage independent growth in vitro. Proc. Natl. Acad. Sci. USA 1975; 72, 4435-9.

[22] W Chamulitrat. Role of gp91phox homolog Nox1 in induction of premalignant spindle phenotypes of HPV 16 E6/E7-immortalized human keratinocytes. Sci. World J. 2010; 10, 1435-49.

[23] J Yang, E Lam, H Hammad,T Oberley and L Oberley. Antioxidant enzyme levels in oral squamous cell carcinoma and normal human oral epithelium. J. Oral Pathol. Med. 2002; 31, 71-7.

[24] F Hecht, JM Cazarin, CE Lima, CC Faria, AAdC Leitão, ACF Ferreira, DP Carvalho and RS Fortunato. Redox homeostasis of breast cancer lineages contributes to differential cell death response to exogenous hydrogen peroxide. Life Sci. 2016; 158, 7-13.

[25] AM Faten, AA Abo-Aziza and AA Zaki. Confluence-associated proliferation and osteogenic differentiation of bone marrow mesenchymal stem cell (BMMSCs). Int. Res. J. Biological Sci. 2016; 5, 44-56.

[26] Q Zhang, N Yu and C Lee. Mysteries of TGF- $\beta$ paradox in benign and malignant cells. Front Oncol. 2014; 4, 94.

[27] ER Butch and KL Guan. Characterization of ERK1 activation site mutants and the effect on recognition by MEK1 and MEK2. J. Biol. Chem. 1996; 271, 4230-5.

[28] YC Hseu, MS Lee, CR Wu, HJ Cho, KY Lin, GH Lai, SY Wang, YH Kuo, KJ Kumar and HL Yang. The chalcone flavokawain B induces G2/M cell-cycle arrest and apoptosis in human oral carcinoma HSC-3 cells through the intracellular ROS generation and downregulation of the Akt/p38 MAPK signaling pathway. J. Agric. Food Chem. 2012; 60, 2385-97.

[29] L Si, L Zheng, L Xu, L Yin, X Han, Y Qi, Y Xu, C Wang and J Peng. Dioscin suppresses human laryngeal cancer cells growth via induction of cell-cycle arrest and MAPK-mediated mitochondrialderived apoptosis and inhibition of tumor invasion. Eur. J. Pharmacol. 2016; 774, 105-17. 